\title{
Design and Simulation of GPON networks over different FBG techniques
}

\author{
Baneet Kaur Bhatia ${ }^{1}$, Er. Manjit Singh ${ }^{2}$ \\ ${ }^{1,2}$ (Electronics and Communication Engineering, GNDU Regional Campus, Jalandhar, India)
}

\begin{abstract}
GPON stands for Gigabit-capable Passive Optical Networks and is used in fiber optic communication channels for establishing an effective communication between source and destination. Digital GPON communication uses various encoding schemes for successful communication but the issue was that most of research work had been conducted by using NRZ encoding scheme which is quite effective encoding schemes. Hence the proposed work implements the FBG, Ideal FBG and Uniform FBG system as the objective of this study is to analyze the performance of NRZ encoding scheme on three different type of FBG systems. . The simulation is done by using optisystem platform and the results are evaluated in the form of $Q$ factor and BER. The result section of this study shows the efficiency of the proposed work.
\end{abstract}

Keywords: Optical Fiber Communication network, Fiber Brag Grating, NRZ

\section{Introduction}

Optical fiber based functional technologies like fiber-to-the home, fiber-to-the-building, etc. Have the recognized benefits of low loss, high bandwidth, and long reach and is presently being deploying globally [1]. In point-to-point and point-to-multipoint time-multiplexed passive optical network (PON) architectures [2], Fiberto-the-home networks are being installed. As shown in fig.1 [3] general passive optical network (PON) which delivers the three-layered services to the customers such as residence, university campus and user grounds from the central office (CO). The central office with the upper limit of $1550 \mathrm{~nm}$ and the lower limit of $1300 \mathrm{~nm}$ is experienced at the receiver end [4]. The two foremost standards for the PONs have emerged the Ethernet PON (EPON) [6] and the gigabits PON (GPON) [7].

A GPON system is a bi-directional point-to multipoint network design disposing optical access lines among a carrier's central office and customer sites [9]. Gigabit Passive Optical Network (GPON) is explained by ITU-T recommendation series G.984.1 using G.984.4. G.984 standard series describe common features of GPON (G.984.1) as well as physical layer specification (G.984.2) transmission layer specification (G.984.3) and Optical Network Unit management and control specification (G.984.4). GPON can transfer not only Ethernet, but ATM and TDM traffic by using GPON encapsulating method (GEM) [10]. GPON is considered as the strongest for the extensive deployments.

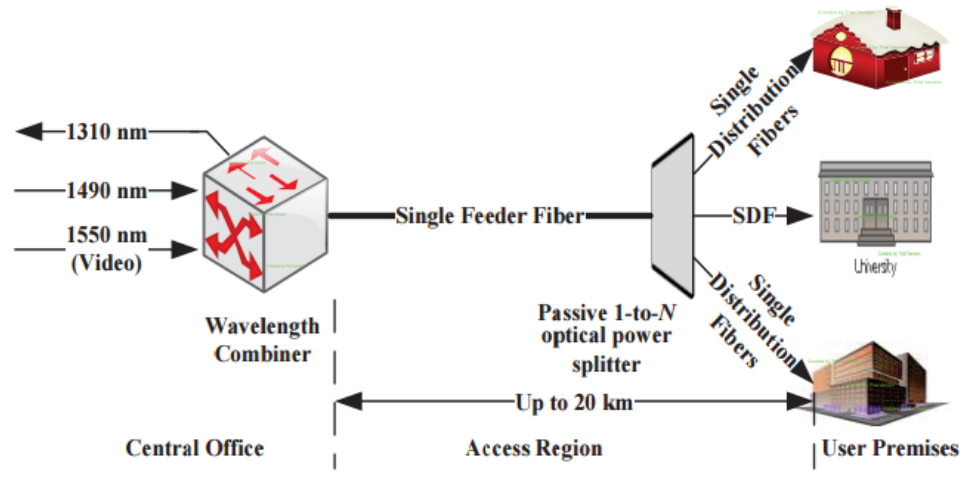

Fig 1 General PON network [3]

\subsection{GPON}

Only Optical Line Termination and Optical Network Unit are contained in Active transmission equipment in GPON network. Figure 2 shows logical network architecture with various FTTx options. 


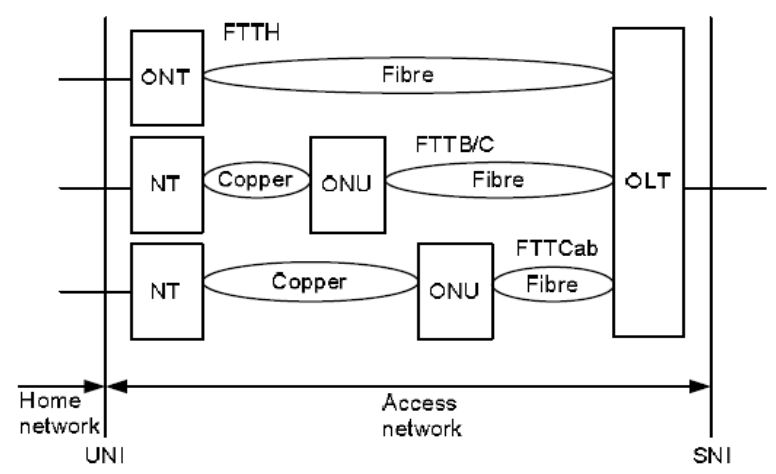

Fig 2 FTTx network Architecture

\subsubsection{Gpon Features}

- Operating wavelength

The operating wavelength range for downstream direction is $1480-1500$ and for upstream direction is 1260 1360. In addition, for downstream RF video distribution the wavelength range $1550-1560 \mathrm{~nm}$ can be used. The wavelength of downstream direction and upstream directions cannot be same.

- Forward error correction

Forward Error Correction is an arithmetical signal-processing method that encrypts data so that errors can be identified and rectified. With FEC, unnecessary information is published along with the unique information. The amount of duplicate information is less so FEC doesn't bring in a lot of overhead.

- Transmission containers

Transmission containers (T-CONT) are used to manage the upstream bandwidth allotment in the GPON. TCONTs are mainly used to surpass the upstream bandwidth use on the GPON. T-CONTs enable Quality of Service (QoS) completion in upstream direction. There are five types of T-CONT. T-CONT 1 ensures fixed bandwidth allocation for time-sensitive applications (VoIP). T-CONT 2 ensures fixed bandwidth allocation for non time-sensitive applications. T-CONT 3 is mix of minimum ensured bandwidth plus additional nonguaranteed. T-CONT 4 is dynamically allocated without any ensured bandwidth. TCONT 5 is mix of all service categories.

- Dynamic bandwidth allocation

Dynamic bandwidth allocation (DBA) is a technique that permits quick adoption of user's bandwidth allotment based on current traffic necessities. DBA is managed by OLT, which allocates bandwidth volume to ONUs. This technique is applicable only in upstream direction

\subsubsection{GPON transmission}

GPON uses GPON Encapsulation Method as a method which enclosed data over GPON. Even though any type of data can be enclosed, actual types dependent on service situation. GEM offers connection-oriented communication.

\section{Problem Formulation}

With the advent in the technology the means of communication also get changed or enhanced. The Optical fiber is one of the examples from the list. Optical fiber is considered as the most advance mean of communication over the network. But as the technology gets advance the requirements of the users also go higher. After having a review to the related work that has been done in this field, it is derived that lot of the work had been done in this field to make the proficient performance of the system. But still there are some backlogs. As the traditional system uses the NRZ encoding mechanism which does not consider the first data bit to be a polarity change. Another issue was related to the used grating techniques as reviewed that most of the traditional works implements or focus the FBG grating technique whereas the other techniques can also be implemented. Hence there is a need to develop such a system or mechanism which eliminates the issues that occur due to the FBG or NRZ coding.

\section{Proposed Work}

After having a review to the research questions that arises after studying the traditional work that was purposely conducted in the field of fiber optics, it is decided to develop such a system which can accomplish the single point to multiple point communication without any problem. Hence the proposed work will implement the NRZ encoding because it is quite effective and reliable encoding scheme used in many research works. And NRZ encoding scheme is analyzed with Simple FBG system, Ideal FBG system and Uniform FBG system. . 


\section{Results}

The proposed GPON model is created by using opti system. The proposed work develops an NRZ encoding based fiber optic system which is capable to perform single point to multiple point communication. The following figures 3,4, and 5 represents the simulation model corresponding to Ideal FBG, Uniform FBG and FBG. The simulation models comprises of Pseudo random BNRZ pulse generator, Bit Error Rate analyzer, Wave laser source, Bessel optical filter and 3R regenerator etc.

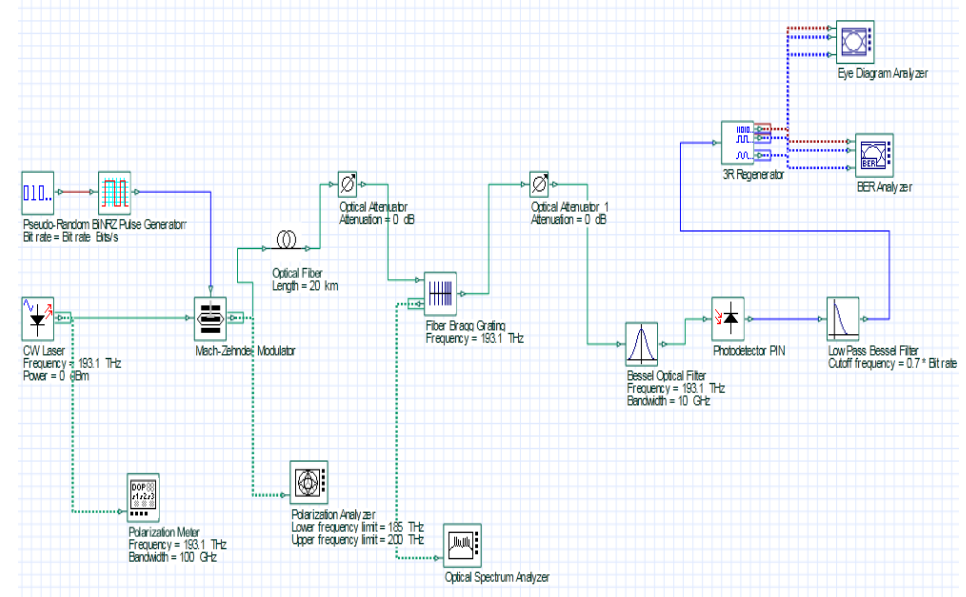

Fig 3 Simulation model (FBG)

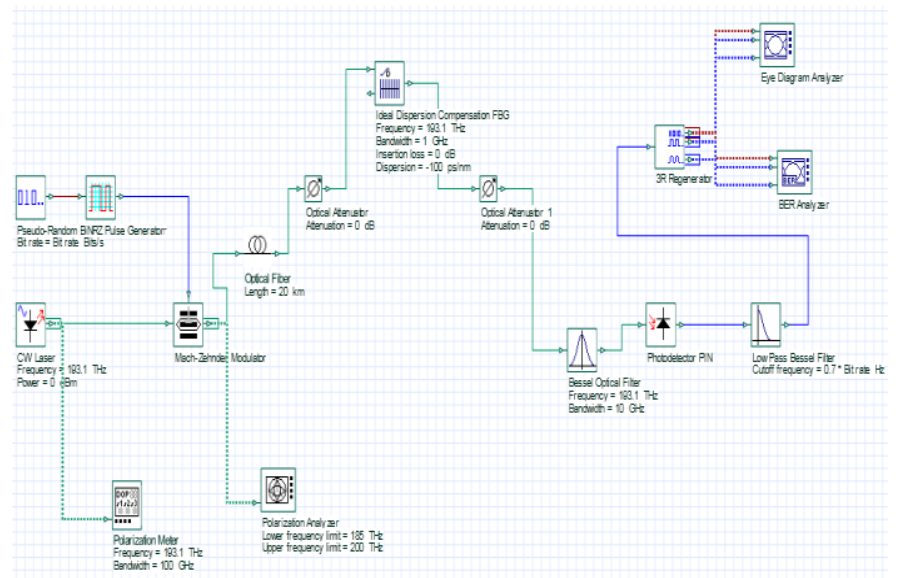

Fig 4 Simulation model (Ideal FBG)

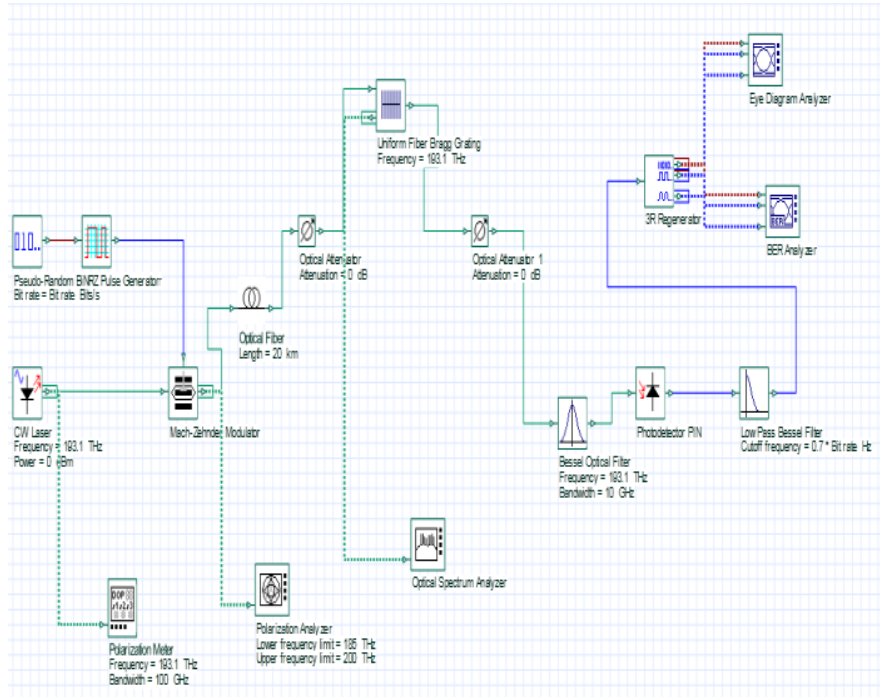

Fig 5 Simulation model (Uniform FBG) 
The following eye diagram represents the maximum quality factor, minimum BER, eye height, threshold for iteration 1, 2 and 3 of FBG techniques respectively. From Eye diagrams corresponding to all iterations of FBG, Ideal FBG and Uniform FBG, table1, 2 and 3 has been derived which shows the values of quality factor and BER corresponding to all three techniques. The figure below represents the BER of the simulink model when input power level is set to $5 \mathrm{dBm}$. The graph below shows the eye diagram along with BER, Quality factor and Threshold value. The value of Q Factor is 4.0399, BER is 2.96878e-005 and threshold value is 0.0002030 .
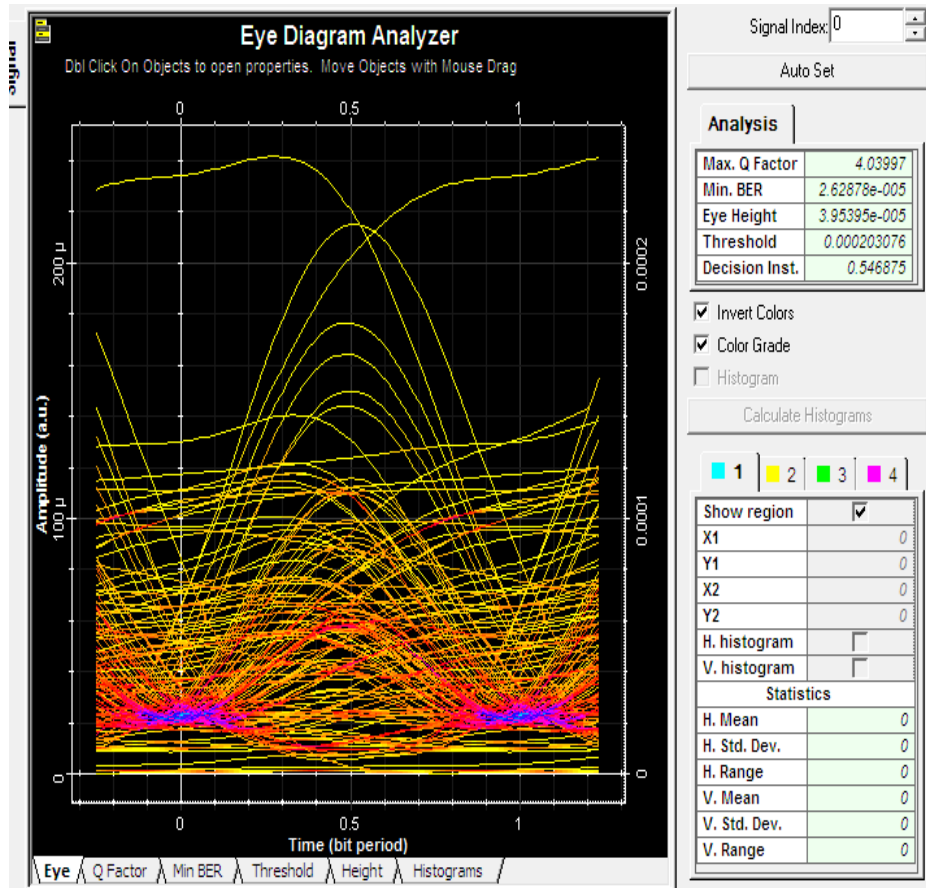

Fig6 Eye Diagram of FBG for Iteration 1

In eye diagram of figure 7 the observed $Q$ factor is 8.70 and BER is $1.14 e^{-018}$

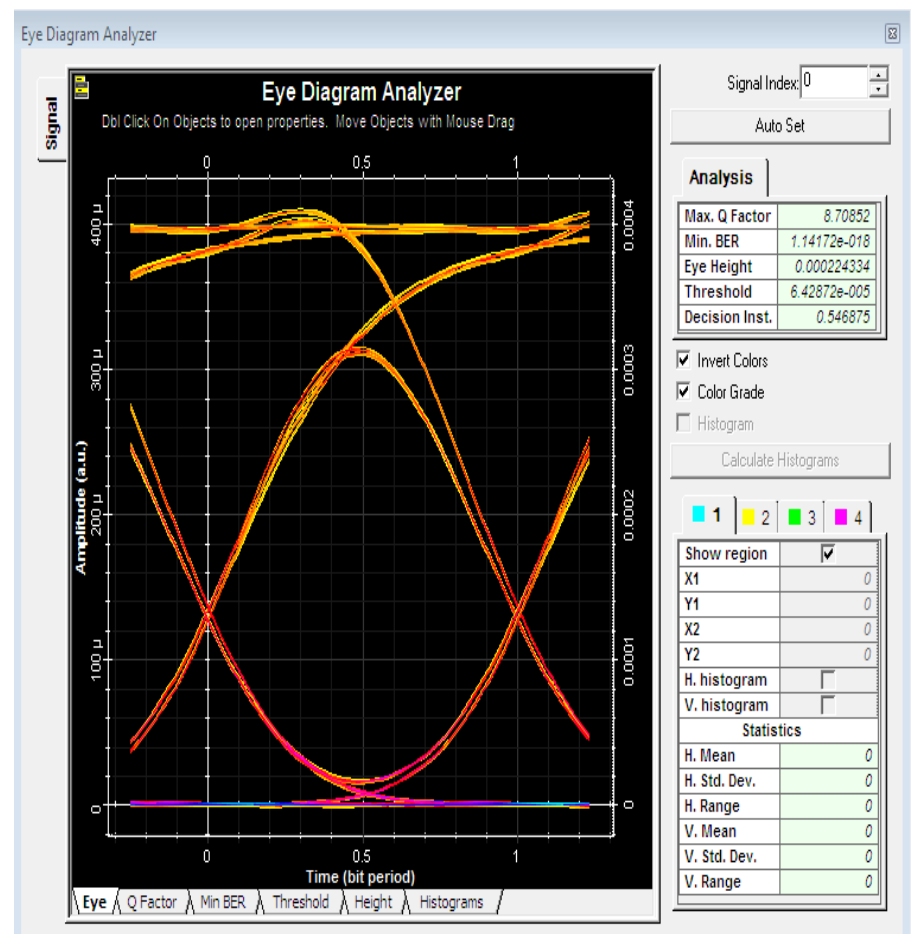

Fig7 Eye Diagram of FBG for Iteration 2 
The BER of eye diagram in figure 8 is $8.56 e^{-019}$ and $\mathrm{Q}$ factor is 8.73 .
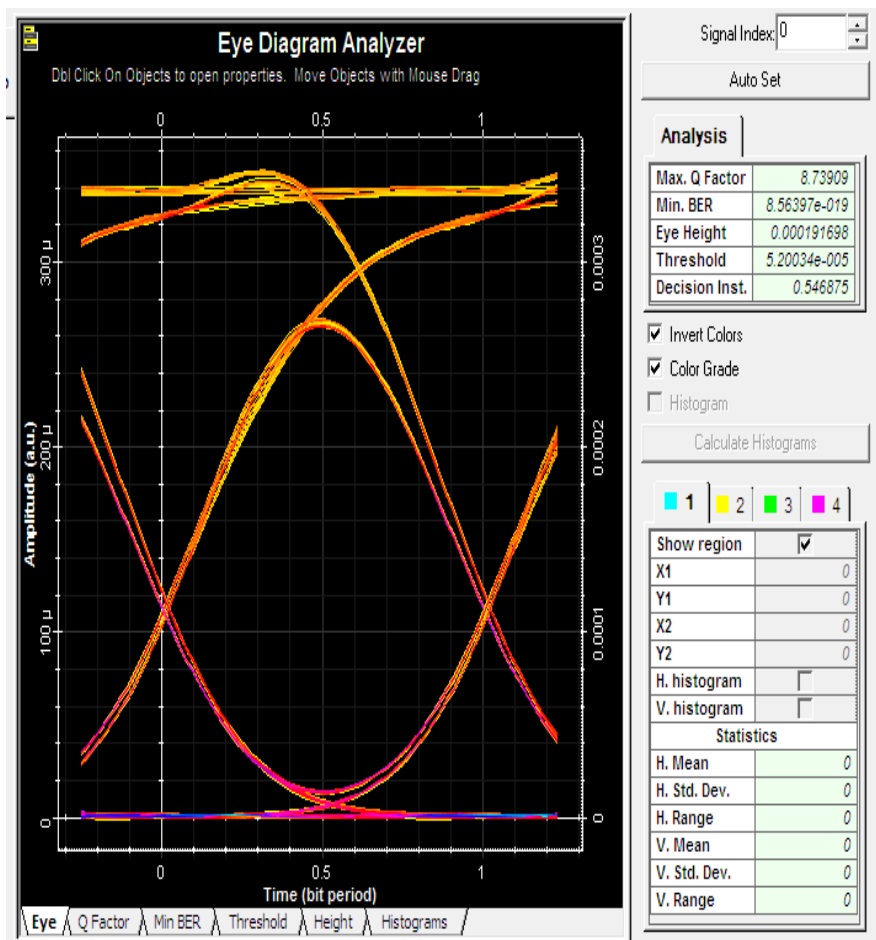

Fig8 Eye Diagram of FBG for Iteration 3

On the basis of above three eye diagrams it is concluded that the BER of the model increases with the increase in input power and correspondingly it leads to the reduction in Q Factor of the simulink model.

Table1 Performance evaluation of FBG

\begin{tabular}{|c|l|l|l|}
\hline S. No. & Iterations & Q. Factor & BER \\
\hline 1. & Iteration 1 & 8.7 & $8.56 e^{-019}$ \\
\hline 2. & Iteration 2 & 8.05 & $3.147 e^{-016}$ \\
\hline 3. & Iteration 3 & 7.49 & $2.65 e^{-014}$ \\
\hline
\end{tabular}

TABLE 1 depicts the performance of FBG with respect to obtained quality factor and BER during all three iterations. The highest $\mathrm{Q}$ factor is observed in first iteration which is 8.7 and minimum BER is in case of iteration 3.

Table2 Performance evaluation of Uniform FBG

\begin{tabular}{|c|l|l|l|}
\hline S. No. & Iterations & Q. Factor & BER \\
\hline 1. & & 8.708 & $1.14 e^{-018}$ \\
\hline 2. & Iteration 1 & & $1.19 e^{-016}$ \\
\hline 3. & Iteration 2 & 8.170 & $2.96 e^{-019}$ \\
\hline
\end{tabular}

TABLE 2 shows the quality factor and BER of uniform FBG. The observed Q factor is 8.708 and BER is 1.114.

Table3 Performance evaluation of Ideal FBG

\begin{tabular}{|c|l|l|l|}
\hline S. No. & Iterations & Q. Factor & BER \\
\hline 1. & Iteration 1 & 4.039 & $2.62 e^{-005}$ \\
\hline 2. & Iteration 2 & 0 & 1 \\
\hline 3. & Iteration 3 & 0 & 1 \\
\hline
\end{tabular}

TABLE 3 shows the values of performance parameters in case of Ideal FBG. And the value of Q factor is evaluated 4.039 and for BER, it is 1 . 


\section{Conclusion And Futurescope}

This work presents an explanation of fiber optical communication system. The Optical fiber communication system attracts lot of users due its various characteristics such as wider bandwidth, flexibility etc. This work gives a proposal for multiuser communication by utilizing NRZ encoding schemes and Fiber Brag Grating system. The simulation is done on three various FBG systems as Simple FBG, Uniform FBG and Ideal FBG. Further research can enhance by applying the present work using wavelength division multiplexing system.

\section{References}

[1] Sanjeev Verma et al, "Performance analysis of Q-Factor and polarization for GPON network using Optisystem", International Conference on Information Technology, Pp. 138-141, 2016

[2] Ivica Cale et al, “Gigabit Passive Optical Network - GPON”, Int. Conf. on Information Technology Interfaces, Pp. 679-684, June 2007

[3] Naresh Kumar, "Improved performance analysis of Gigabit passive optical networks", Optik 125, Pp. 1837-1840, September 2013

[4] Marilet De Andrade et al, "Passive optical network (PON) supported networking", Optical switching and networking, Vol. 14, No. 1, Pp. 1-10, August 2014

[5] Marino Rodrigues et al, "Plastic Optical Fibers in Access Networks", Conference on Electronics, Telecommunications and Computers, Procedia Technology, Vol. 17, pp. 601-607, 2014

[6] Bhavin J. Shastri et al, "Effect of channel impairments on the performance of burst-mode receivers in gigabit PON", Circuits and Systems, September 2008

[7] D. Verhulst et al, "Upstream experiments on the gigabit PON physical medium layer", Optical Fiber Communication, March 2005

[8] Lukas Malina, "Security solution with signal propagation measurement for Gigabit Passive Optical Networks", Optik - International Journal for Light and Electron Optics, Vol. 127, No. 16, Pp. 6715-6725, August 2016

[9] Sumanpreet et al, "A review on Gigabit Passive Optical Network (GPON)", International Journal of Advanced Research in Computer and Communication Engineering, Vol. 3, No. 3, March 2014

[10] Debasmita Hazra et al, "Performance Analysis of 1.25 Gbps Downstream transmission of GPON-FTTX", International Journal of Scientific Engineering and Research, Vol. 4, No. 3, Pp. 1-7, March-2013

[11] Sanjeev Dewra et al, "Fabrication and Applications of Fiber Bragg Grating- A Review", Advanced Engineering Technology and Application, No. 2, Pp. 15-25, 2015

[12] Sanjeev Verma et al, "Performance analysis of GPON network for maximum Q-FACTOR and minimum BER for various fiber length using uniform fiber Bragg's grating", Information Technology (InCITe) - The Next Generation IT Summit on the Theme Internet of Things: Connect your Worlds, International Conference on, February 2017

[13] Lin Qi et al, "Research of a Novel Dispersion Compensator Based on Uniform Fiber Bragg Grating", Wireless Communications, Networking and Mobile Computing, October 2009

[14] Christi K. Madsen et al, "Optical Filter Design and Analysis: A Signal Processing Approach”, 1999 A

[15] Nguyen Dong-Nhat et al, "NRZ versus RZ over Absolute Added Correlative coding in optical metro-access networks", Optical Communications, Vol. 387, Pp. 30-36, March 2017

[16] Fadil Paloi et al, "Optimisation of dispersion compensating in a long-haul fibre for RF transmission of up to $100 \mathrm{Gbit} / \mathrm{s}$ by using RZ and NRZ formats", Optik- International Journal for Light and Electron Optics, Vol. 131, Pp. 640-654, February 2017

[17] Richa Bhatia et al, "Performance improvement for $N \times 80-\mathrm{Gb} / \mathrm{s}$ WDM transmission link with optimized alternate polarization", Optik - International Journal for Light and Electron Optics, Vol. 124, No. 23, Pp. 6013-6016, December 2013

[18] Lanlan Li et al, "Reconfigurable all-optical logic gate using four-wave mixing (FWM) in HNLF for NRZ-PolSK signal", Optics Communications, Vol. 283, No. 19, pp. 3608-3612, October 2010

[19] Monika Mehra et al, "Performance analysis of coherent optical communication system for higher order dual polarization modulation formats", Optik - International Journal for Light and Electron Optics, Vol. 135, Pp. 174-179, April 2017

[20] J.C. Cartledge et al, "The effect of chirping-induced pulse shaping on the performance of 11 Gbit/s lightwave systems", IEEE Photonics Technology Letters, Vol. 1, No. 10, Pp. 346-348, Oct. 1989

[21] Harmanjot Singh et al, "Analysis of Advance Modulation Format Using Triple Play Services in WDM/TDM PON and Scaling Technique: A Review", International Journal of Advanced Research in Computer Science, Vol. 8, No. 3, Pp. 826-830, April 2017

[22] Affida M. Zin et al, "The Characterization of Radio-over-Fiber Employed GPON Architecture for Wireless Distribution Network", International Journal of Machine Learning and Computing, Vol. 1, No. 5, Pp. 522-527, December 2011

[23] Simranjeet Singh et al, "Comparison of Advance Data Modulation Formats in $4 \times 10$ Gbps WDM Optical Communication System using YDFA, EDFA and Raman Amplifier”, International Journal of Innovations in Engineering and Technology (IJIET), Vol. 5, No. 2, Pp. 389-396, April 2015

[24] Bhumit P. Patel et al, "COMPARISON OF DIFFERENT MODULATION FORMATS FOR 8 CHANNEL WDM OPTICAL NETWORK AT 40 GBPS DATARATE WITH NON-LINEARITY”, International Journal of Advanced Research in Engineering and Technology (IJARET), Vol. 5, No. 2, February 2014

[25] Ali Israr et al, "Performance Analysis of Advance Optical Modulation Formats for GPON System", International Conference on Frontiers of Information Technology, Pp. 77-80, December 2015 